\title{
Path Analysis of the Association Between Socio-economic Status, Anxiety, Perceived Stress, Social Support and Women's Depression
}

\author{
Roshanak Vameghi $^{1}$, Sedigheh Amir Ali Akbari ${ }^{*}{ }^{\mathbb{D}}$, Homeira Sajjadi $^{3}$, Firoozeh Sajedi ${ }^{1}$, Hamid Alavimajd $^{4}$
}

\begin{abstract}
Objectives: Given the importance and prevalence of depression among women, this study aimed to test the correlation model between socio-economic status, anxiety, perceived stress, social support and women's depression in reproductive age using path analysis.

Materials and Methods: This analytical cross-sectional study was conducted on 1065 women of reproductive age selected randomly from clinics affiliated to Shahid Beheshti University of Medical Sciences, Tehran, Iran. Data were collected using demographic and socio-economic status questionnaires, the perceived stress and the social support Scales, Spielberger's Anxiety Inventory and Beck's Depression Inventory. The data were analyzed using SPSS version 19.0 and LISREL version 8.8.

Results: The final path model fitted well (GFI=1; RMSEA $=0.09)$ and showed that socioeconomic status had direct $(\beta=-0.22)$ and indirect effect $(\beta=-0.0645)$, perceived stress had direct effect $(\beta=0.22)$, social support had direct $(\beta=-0.21)$ and indirect effect $(\beta=-$ $0.033)$, anxiety had direct effect $(\beta=0.18)$ on depression, and overall, socioeconomic status had the greatest effects on depression $(\beta=-0.2845)$.

Conclusions: According to the obtained results, screening for the examined variables is recommended to prevent and diagnose depression and promote health in women.

Keywords: Socioeconomic status, Psychological factors, Depression, Social Support, Perceived Stress, Anxiety.
\end{abstract}

\section{Introduction}

Depression is one of the most common mental health disorders. There are currently 300 million people worldwide affected by this disease while half of them are not receiving any treatment. Depression is anticipated to become the second leading cause of disabilities in the world by 2020 (1-3). Depressed people have a lower quality of life (4). Depression causes deterioration in interpersonal relationships (5). Previous studies have shown that depression is associated with gender, age, marital status, and other demographic factors. Women are 2 to 3 times at higher risk of developing depression than men (6-8). The lifetime risk of depression is $5 \%$ to $10 \%$ among men and $10 \%$ to $25 \%$ among women (9).

Many factors from puberty to menopause, e.g. genetic predisposition, hormonal fluctuations, side effects of delivery, environmental stressors, differences between psychological and social pressures and learned behavior patterns related to distress, and women's reactions to stress, might be involved in the depression in women (10-12). History of child abuse, being single, widowed, or divorced, having more than 3 children, smoking, alcohol and drug abuse, poverty, lower age, undesirable employment status, low education, and stressors (such as conflicts and disputes with the spouse) are correlated with women's depression (13).

Stress is a key factor in many psychosocial problems and has a permanent presence in all aspects of life in varying degrees. Physiological changes caused by stress can disrupt activities of the body system, and thus pave the way for physical and psychological diseases $(14,15)$. The incidence of depression is associated with individuals' perceived stress. Furthermore, stress has a major role in progress and exacerbation of depression and anxiety disorders (16).

Based on recent evidence, mental health is related to low social support (17). Senturk et al in 2011 found that women with high depression had lower social support (18). Moreover, studies on social support have confirmed positive and beneficial effects of close interpersonal relationships (19). Social support has a significant effect on the quality of life (20) and can alleviate the harmful effects of stressors on the immune system (21). In fact, a person's reaction to stress may be less severe in the 
presence of his/her friends and acquaintances than when they face the stressful conditions alone (22).

Poor socioeconomic status affects health in the course of life. People in lower social strata are often twice more likely to suffer from illnesses than those in upper classes. Not only is physical health affected by socioeconomic status, but also mental health is associated with the individual's social class, therefore, poorer classes have greater odds of developing psychological disorders (23). Furthermore, women with low income and difficult jobs experience more stress (24). Depression and psychological disorders are observed more frequently in impoverished women (25).

Caring about mental health is important and conducting research on women's mental health should be particularly prioritized (26). Symptoms of depression are generally present in about $50 \%$ of patients with major depressive disorder before the first episode of the disease is diagnosed. Therefore, early diagnosis and treatment of primary symptoms of depression would prevent severe depression (27).

Fortunately, "path analysis" a well-suited statistical method for mental health or other longitudinal studies where phenomena do not have single cause, but are the product of chains of predisposing influences, which involve many complex interactions and where the causal direction between variables is ambiguous allows us to try to unveil this ambiguity and complexity to a certain degree (28).

This study used path analysis to clarify the relationships between depression and socioeconomic status, anxiety, social support, and perceived stress in women of reproductive age (Figure 1 ).

\section{Materials and Methods}

This analytical cross-sectional study was conducted on 1065 women who attended health centers affiliated to Shahid Beheshti University of Medical Sciences, Tehran, Iran during 2014. After being approved by the Ethics Committee of Social Welfare and Rehabilitation University, a list of the mentioned clinics in various regions of the city was prepared. Some centers were then randomly selected

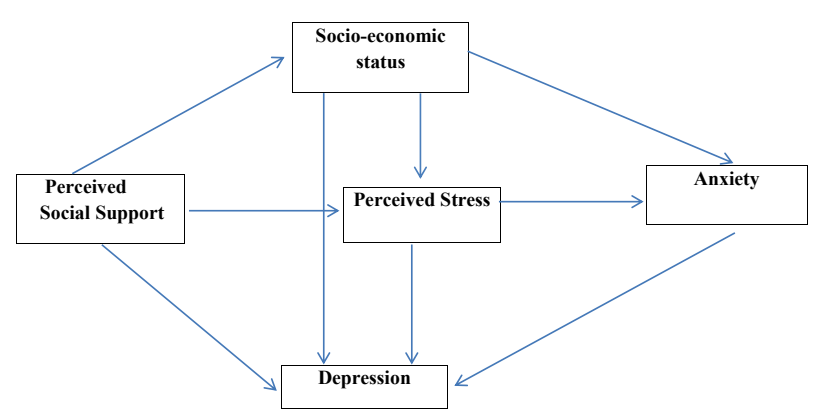

Figure 1. The Theoretical Path Model for the effects of Socioeconomic Status, Anxiety, Perceived Stress, Social Support on Women' Depression from each region and the sample size was determined based on the population of each center. The participants were provided with details about the study objectives and asked to sign an informed consent form. 18-35-year-old Iranian women who had no medical illness and no history of mental disorders (reported by themselves and their family members) were recruited.

The data collection tools used included:

Demographic information questionnaire and the Socioeconomic Inventory:

The demographic information questionnaire used was researcher-designed and 10 faculty members confirmed its face and content validity. It included such demographic factors as the woman's and her husband's age, the woman's and her husband's level of education, the woman's employment status and so on. The socioeconomic status of the subjects was assessed using the Socio-economic Inventory designed by Garmaroudi et al in 2010 (29), with components including the subject's level of education, the spouse's level of education, ratio of home area to household size, price of the home per square meter, facilities and amenities (such as car and computer ownership) and family income. The correlation between these factors and the total score obtained in the inventory has been reported to be 0.87 , and the test-retest reliability has been determined to be 0.96 . The inventory provides a cut-off point of 16 for differentiating between favorable and unfavorable socioeconomic status. The maximum obtainable score is 48 .

\section{Spielberger's Anxiety Inventory}

The State-Trait Anxiety Inventory (STAI) is an introspective psychological inventory consisting of 40 self-report items pertaining to anxiety. The anxiety scores obtained in this inventory range from a minimum of 20 to a maximum of 80, with scores of 20-40 indicating mild anxiety, 41-60 indicating moderate anxiety and 61-80 indicating severe anxiety. Numerous studies have determined the validity and reliability of inventory for measuring anxiety (30-32). The reliability of this inventory has been examined in 2 studies in Iran; one study conducted in Tehran calculated it as 0.91(33), and another study conducted in Mashhad calculated it as 0.95 (34). The present study calculated the test-retest reliability of the inventory as 0.94 .

Cohen's Perceived Stress Scale

Cohen's scale for the assessment of perceived stress is developed to measure perceived stress in the preceding month (35) and is widely used in different countries. It has been translated into different languages and standardized for use in different cultures. The present study used the 14 -item version of the scale. The score obtained in this scale varies between 0 and 56 and higher scores indicate a higher degree of perceived stress. No cut-off points have been specified for this scale. Bastani et al in 2008 
determined the reliability of the Persian version of the scale through measuring its internal consistency and calculated its Cronbach's alpha as 0.74 (36). Other studies using this scale in Iran have calculated its Cronbach's alpha as 0.840.86 (37-39). The present study calculated the reliability (internal consistency) of the scale as 0.88 and its test-retest reliability as 0.92 .

\section{Perceived Social Support Inventory}

The Perceived Social Support Inventory was designed by Sarason et al in 1983 (40) and was translated into Persian by Nasseh et al. The validity and reliability of the inventory were measured and its internal consistency was confirmed with a Cronbach's alpha of 0.95 (41). Other studies conducted in Iran have calculated the reliability of the inventory as $0.86-0.89(38,42)$. This inventory has also been used in studies conducted in other countries $(43,44)$. The minimum and maximum scores that can be obtained in this inventory are 12 and 84, respectively. The present study calculated the reliability (internal consistency) of the scale as 0.89 and its test-retest reliability as 0.92 .

\section{Beck's Depression Inventory}

Beck's Depression Inventory has 21 items with a score ranging from 0 to 63 . Different studies have confirmed the reliability of this inventory $(45,46)$. It has also been standardized for use in Iran, the internal consistency of the inventory was confirmed for use in Iran with a Cronbach's alpha of 0.87 and its reliability was then calculated to be 0.74 (47). The present study calculated the test-retest reliability of the inventory as 0.92 .

\section{Statistical Analysis}

The model was tested through LISREL version 8.8 for the path analysis and SPSS version 19.0 (Chicago, IL, USA) using the Mann-Whitney test, the chi-square test and the independent $t$ test at a significance level of 0.05 .

\section{Results}

There was no significant difference in the mean age between depressed and non-depressed women. However, the 2 groups had significant differences in terms of women's and their husbands' education levels (Table 1).

Different levels of depression (mild to severe) were found in $47.69 \%$ of the studied women. The participants' mean stress score was $24.23 \pm 7.92$. Most participants (44.2\%) enjoyed moderate levels of social support. The mean scores of spousal support, family support, and support from friends were $24.62 \pm 5.49,23.70 \pm 5.65$, and $18.66 \pm 7.55$, respectively. The majority of the women $(72 \%)$ had favorable economic conditions. However, $74 \%$ of the participants had moderate to severe anxiety (Table 2). Correlation between socio-economic status, anxiety, perceived stress, social support and women' depression is shown in Table 3.

Table 4 presents direct, indirect and the overall effects of the parameters mentioned on depression. The indices GFI, CFI, and RMSEA were used to investigate the model fitness (Table 5). According Figure 2 the final path model fitted well.

\section{Discussion}

Results showed that the proposed model has a good fit. Our findings showed that socioeconomic and social support had both direct and indirect relationships with depression. Moreover, perceived stress and anxiety were directly correlated with depression in women. Socioeconomic status had the greatest effect on depression.

In this study, $47.69 \%$ of women suffered from mild to severe depression. Previous studies in Iran reported the rate of depression as $21.5 \%$ to $54.7 \%(48,49)$. Aeenparast et al reported the prevalence of severe and very severe depression as $8.3 \%$ (50). Ahmadvand et al estimated this rate as $13.5 \%$ (51). Differences in sample size and instruments used to assess depression might have been responsible for the different rates reported by previous

Table 1. Comparison of the Demographic Characteristics in Depressed and Non-depressed Women

\begin{tabular}{|c|c|c|c|}
\hline \multirow{2}{*}{ Variables } & \multicolumn{2}{|c|}{ Groups } & \multirow{2}{*}{$\boldsymbol{P}$} \\
\hline & Depressed women, $n=508$ & Non-depressed women, $n=557$ & \\
\hline Women's age (mean $\pm S D$ ) & $28.74 \pm 4.52$ & $28.52 \pm 4.77$ & 0.312 \\
\hline Husband's age (mean \pm SD) & $32.19 \pm 4.42$ & $32.48 \pm 4.58$ & 0.324 \\
\hline Women's education, No. \% & & & $<0.001$ \\
\hline Primary & 55 (10.9) & $48(8.6)$ & \\
\hline High school & $296(58.3)$ & $301(57.1)$ & \\
\hline Diploma & 142 (27.9) & 183 (32.9) & \\
\hline College & 15 (2.9) & $25(4.4)$ & \\
\hline Husband's education, No. \% & & & $<0.001$ \\
\hline Primary & $47(9.3)$ & $49(8.8)$ & \\
\hline High school & $280(55.1)$ & $282(50.6)$ & \\
\hline Diploma & $164(32.3)$ & $187(33.6)$ & \\
\hline College & $17(3.3)$ & $39(7)$ & \\
\hline Employment & & & 0.123 \\
\hline Unemployed (Housewife) & $471(92.7)$ & $515(92.5)$ & \\
\hline Employed & $37(7.3)$ & $42(7.5)$ & \\
\hline
\end{tabular}


Table 2. Mean $\pm S D$, Minimum and Maximum Scores of Socioeconomic Status, Anxiety, Perceived Stress, Social Support and Women' Depression

\begin{tabular}{lcccc}
\hline & Mean & SD & Min value & Max value \\
\hline Socio-economic status & 20.89 & 6.14 & 8 & 47 \\
Anxiety & 44.65 & 5.83 & 22 & 72 \\
Perceived stress & 24.23 & 7.92 & 0 & 44 \\
Perceived social support & 60.58 & 14.09 & 12 & 84 \\
Depression & 12.62 & 4.79 & 2 & 74 \\
\hline
\end{tabular}

research.

In our study, socioeconomic status affected depression both directly and indirectly (through its effects on social support and perceived stress).

Miech and Shanahan found depression to be correlated with social support, income level, and socioeconomic status (52). Kosidou et al indicated that income level was correlated with the incidence of depression among women (53). Women with low economic status were 3.57 times more likely to develop depression (54). Aeenparast et al reported relationships between depression and women's employment status and educational level, as indicators of economic status, i.e. depression was more prevalent among working women (50). Women with lower income have to deal with greater levels of stress, which can be a predictor of mental health status (55).

In our study, social support was directly correlated with depression. Tomczak-Witych observed a significant difference in received social support between depressed and non-depressed women (56). However, Ezzati et al confirmed a relationship between family support and the incidence of depression and failed to find any relationships between other types of support and depression (57).

Researchers have focused on 2 processes in their efforts to determine the effects of social support on health. The first process involves the direct effect of social support on health and indicates that the presence of support or its absence (e.g. in cases of social isolation) directly affects people's health. The second process acts through what is called the "moderating effect". It suggests that rather than exerting direct effects on health, social support moderates the effects of acute and chronic stresses on human health. It has long been known that dealing with various stressors, such as stressful events, may threaten the health of some (not all) individuals. It is thus assumed that the causal effect of life events on the incidence of diseases is moderated by supportive factors such as social support (58).
Table 4. Path Coefficients for Socioeconomic Status, Anxiety, Perceived Stress, Social Support and Women' Depression

\begin{tabular}{lcccc}
\hline \multirow{2}{*}{ Predictor Variables } & \multicolumn{3}{c}{ Effect ( Standardized $\boldsymbol{\beta}$ ) } & T \\
\cline { 2 - 4 } & Direct & Indirect & Total & Value \\
\hline Socioeconomic status & -0.22 & -0.06455 & -0.28455 & -6.54 \\
Anxiety & 0.18 & - & 0.18 & 5.58 \\
Perceived Stress & 0.22 & - & 0.22 & 6.58 \\
Perceived Social support & -0.21 & -0.033 & -0.243 & 6.15 \\
\hline
\end{tabular}

Table 5. Goodness of Fit Indices for the Model

\begin{tabular}{lllllll}
\hline $\boldsymbol{x} 2$ & $d f$ & $\boldsymbol{P}$ & NFI & CFI & GFI & RMSEA \\
\hline 7.04 & 1 & 0.007 & 0.97 & 0.98 & 1 & 0.09 \\
\hline
\end{tabular}

In the present study, social support was indirectly related to depression (through its effects on stress and anxiety). In other words, women with lower levels of social support were more affected by stress and hence were more likely to develop depression. Social support and relationships have health protective effects and can significantly promote health. Supportive relationships can cause healthier behaviors. People with lower levels of social support are more vulnerable to mental and physical problems (59).

Wang et al found that people with lower levels of social support suffer from greater levels of stress and depression (60). Depending on various personal and social factors, stressful events can cause different reactions in people. Social support has a major role in this regard and acts as an important barrier against depression. It can serve as a positive intervention in coping with major stresses affecting people's health. In other words, social support can facilitate psychological adjustment in people who face major health stresses and reduce the effects of psychological stress by enhancing the person's understanding of stressful events and minimizing the complications of an unpleasant experience (57). He et al reported that social support plays a mediating role in the relationship between isolation and the incidence of depression (61). Spoozak et al concluded that the social support received from family and friends could reduce depression (62). Higher incidence of depression and anxiety was reported among the individuals with lower social support. They indicated that social support had a moderating role in the relationship between stress and the incidence of depression (60).

Table 3. Correlation Between Socio-economic Status, Anxiety, Perceived Stress, Social Support and Women' Depression

\begin{tabular}{|c|c|c|c|c|c|}
\hline & Socioeconomic Status & Anxiety & Perceived Stress & Perceived Social Support & Depression \\
\hline Socio-economic status & 1 & $-0.146 * *$ & $-0.096 * *$ & $0.184 * *$ & $-0.249 * *$ \\
\hline Anxiety & & 1 & 0.035 & $0.090 * *$ & $0.181^{* *}$ \\
\hline Perceived stress & & & 1 & $-0.105^{* *}$ & $0.184 * *$ \\
\hline Perceived Social support & & & & 1 & $-0.315 * *$ \\
\hline Depression & & & & & 1 \\
\hline
\end{tabular}

**Correlation is significant at 0.01 level (2-tailed). 


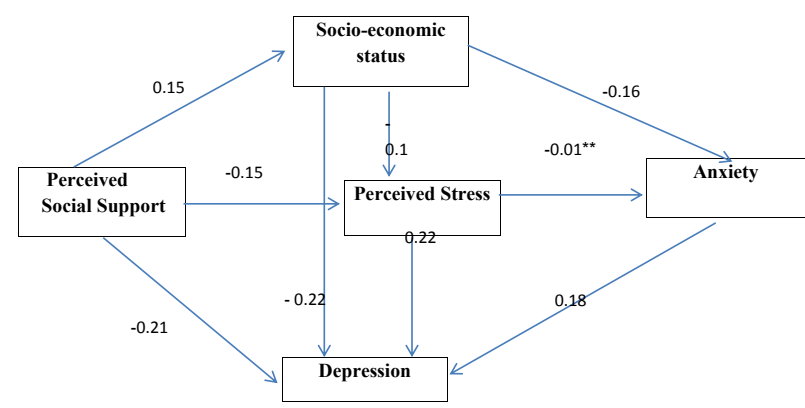

Figure 2. Full Empirical Model (empirical path model for the effects of Socio-economic Status, Anxiety, Perceived Stress, Social Support on Women' Depression [standardized $\beta$ was showed in model]).

In this study, stress and anxiety were directly correlated with depression. Two major physiological processes occur when a person faces stressors, such as needs and threats. First, the autonomic nervous system is activated and catecholamine, especially norepinephrine and epinephrine, are released. During the second process, the hypothalamic-pituitary-adrenal axis is activated through the release of the corticotrophin-releasing hormone, adrenocorticotropic hormone (ACTH), and cortisol. These 2 mechanisms cause not only physiological responses, but also changes in behavior, e.g. loss of appetite, reduced sexual activity, and increased levels of depression, anxiety, and irritability $(63,64)$.

According to Kader Maiden et al, women exposed to high levels of stress were 4.9 times more at risk of depression (65). Severe and prolonged stress can affect a person's ability to adapt, cause physical damage to the body, destroy the joy of life, and cause depression (66). Mehnert et al reported a relationship between anxiety and depression (67). Hammen also confirmed a relationship between depression and anxiety (68).

\section{Conclusions}

Based on our findings, depression had significant relationships with factors such as socioeconomic status, social support, stress, and anxiety. Since depression is a major mental health issue, especially among women, screening for early diagnosis and treatment of the condition seems necessary.

\section{Conflict of Interests}

Authors declare that they have no conflict of interests.

\section{Ethical Issues}

The Ethics Committee (CodeUSWR.REC.1393.152) of Social Welfare and Rehabilitation University approved the study.

\section{Financial Support}

This study was supported by University of Welfare and Rehabilitation Sciences.

\section{Acknowledgements}

The authors are grateful to the managers and personnel of the selected clinics and the mothers who participated in the study.

\section{References}

1. World Health Organization. Depression: A global public health concern. Geneva, Switzerland: WHO; 2012.

2. Ahmadi J, Galal Ahmed M, Ali Bayoumi F, Abdul Moneenum A, Alshawa H. Mental health of dubai medical college students. Iran J Psychiatry Behav Sci. 2012;6(2):7983.

3. Wolniczak I, Caceres-DelAguila JA, Maguina JL, BernabeOrtiz A. Fruits and vegetables consumption and depressive symptoms: A population-based study in Peru. PLoS One. 2017;12(10):e0186379. doi:10.1371/journal.pone.0186379

4. Abbaszadeh F, Kafaei Atrian M, Masoudi Alavi N, Bagheri A, Sadat Z, Karimian Z. Relationship between quality of life and depression in pregnant women. Nurs Midwifery Stud. 2013;2(2):193-197.

5. Sutter-Dallay AL, Murray L, Dequae-Merchadou L, Glatigny-Dallay E, Bourgeois ML, Verdoux H. A prospective longitudinal study of the impact of early postnatal vs. chronic maternal depressive symptoms on child development. Eur Psychiatry. 2011;26(8):484-489. doi:10.1016/j.eurpsy.2010.05.004

6. Ali NS, Azam IS, Ali BS, Tabbusum G, Moin SS. Frequency and associated factors for anxiety and depression in pregnant women: a hospital-based cross-sectional study. ScientificWorldJournal. 2012;2012:653098. doi:10.1100/2012/653098

7. Van de Velde S, Bracke P, Levecque K. Gender differences in depression in 23 European countries. Cross-national variation in the gender gap in depression. Soc Sci Med. 2010;71(2):305-313. doi:10.1016/j.socscimed.2010.03.035

8. Kessler RC, Bromet EJ. The epidemiology of depression across cultures. Annu Rev Public Health. 2013;34:119-138. doi:10.1146/annurev-publhealth-031912-114409

9. Beck AT, Alford BA. Depression: causes and treatments. Philadelphia: University of Pennsylvania Press; 2009.

10. Steiner M, Dunn E, Born L. Hormones and mood: from menarche to menopause and beyond. J Affect Disord. 2003;74(1):67-83.

11. Sajadi H, Vameghi M, Forozan A, Rafiey H, Mohgheghee Kamal SH, Nosrat Abadi M. Prevalence, risk factors and interventions of depression during pregnancy in Iranian Studies (1997-2011). Journal of North Khorasan University of Medical Sciences. 2013;5(2):521-530.

12. Zammit S, Owen MJ. Stressful life events, 5-HTT genotype and risk of depression. Br J Psychiatry. 2006;188:199-201. doi:10.1192/bjp.bp.105.020644

13. Edge D. Ethnicity, psychosocial risk, and perinatal depression--a comparative study among inner-city women in the United Kingdom. J Psychosom Res. 2007;63(3):291295. doi:10.1016/j.jpsychores.2007.02.013

14. Dolatian M, Mirabzadeh A, Forouzan AS, et al. Correlation between self-esteem and perceived stress in pregnancy and ways to coping with stress. Pajoohande. 2013;18(3):148155.

15. Shamsi M, Bayati A, Jahani F, Farhangnia L. The effect of 
Holy Quran recitation on perceived stress among personnel of Arak University of Medical Sciences. Daneshvar Medicine. 2011;19(95):35-44.

16. Yoon KL, Joormann J. Stress reactivity in social anxiety disorder with and without comorbid depression. J Abnorm Psychol. 2012;121(1):250-255. doi:10.1037/a0025079

17. Ceballo R, McLoyd VC. Social support and parenting in poor, dangerous neighborhoods. Child Dev. 2002;73(4):1310-1321.

18. Senturk V, Abas M, Berksun O, Stewart R. Social support and antenatal depression in extended and nuclear family environments in Turkey: a cross-sectional survey. BMC Psychiatry. 2011;11:48. doi:10.1186/1471-244x-11-48

19. Marmot MG, Wilkinson RG. Social determinants of health. USA: Oxford University Press; 2006.

20. Alipour F, Sajadi H, Forouzan A, Nabavi H, Khedmati E. The Role of Social Support in the Anxiety and Depression of Elderly. Iranian Journal of Ageing. 2009;4(11):53-61.

21. Marmot M, Allen J, Bell R, Bloomer E, Goldblatt P. WHO European review of social determinants of health and the health divide. Lancet. 2012;380(9846):1011-1029. doi:10.1016/s0140-6736(12)61228-8

22. Bovier PA, Chamot E, Perneger TV. Perceived stress, internal resources, and social support as determinants of mental health among young adults. Qual Life Res. 2004;13(1):161170. doi:10.1023/B:QURE.0000015288.43768.e4

23. Walsh M, Stephens P, Moore S. Social Policy and Welfare. Pub: Nelson Thornes, UK: 2000.

24. Baheiraei A, Bakouei F, Mohammadi E, Montazeri A, Hosseni M. The Social Determinants of Health in Association with Women's Health Status of Reproductive Age: A Population-Based Study. Iran J Public Health. 2015;44(1):119-129.

25. Petterson SM, Albers AB. Effects of poverty and maternal depression on early child development. Child Dev. 2001;72(6):1794-1813.

26. Lowdermilk DL, Perry SE. Maternity and women's health. Philadelphia: Lippincott Williams \& Wilkins; 2005:2-4.

27. Sadock BJ, Kaplan HI, Sadock VA. Kaplan and Sadock's Synopsis of Psychiatry: Behavioral Sciences/Clinical Psychiatry. Philadelphia: Wolter Kluwer/Lippincott Williams \& Wilkins; 2007.

28. Olobatuyi ME. A user's guide to path analysis. Maryland: University Press of America; 2006:60.

29. Garmaroudi G, Moradi A. Socio-economic status in Iran: A study of measurement index. Payesh. 2010;9(2):137-144.

30. Court H, Greenland K, Margrain TH. Measuring patient anxiety in primary care: Rasch analysis of the 6-item Spielberger State Anxiety Scale. Value Health. 2010;13(6):813-819. doi:10.1111/j.1524-4733.2010.00758.x

31. Nasiri Amiri, F, Salmalian H, Hajiahmadi M, Ahmadi AM. Association between Prenatal Anxiety and Spontaneous Preterm Birth. J Babol Univ Med Sci. 2009;11(4):42-48.

32. Zhang J, Gao Q. Validation of the trait anxiety scale for statetrait anxiety inventory in suicide victims and living controls of Chinese rural youths. Arch Suicide Res. 2012;16(1):8594. doi:10.1080/13811118.2012.641440

33. Kaviani H, Ahmadi Abhari A. Prevalence of Anxiety Disorders in Tehran City. Iranian Journal of Psychiatry and Clinical Psychology. 2003;8(3):4-11.

34. Mahram B. The normative of Spielberger anxiety test in Mashhad city [Thesis]. Tehran: Psychology College Allameh Tabatabaie University; 2000.

35. Cohen S, Kamarck T, Mermelstein R. A global measure of perceived stress. J Health Soc Behav. 1983;24(4):385-396.

36. Bastani F, Rahmatnejad L, Jahdi F, Haghani H. Breastfeeding Self Efficacy and Perceived Stress in Primiparous Mothers. Iran Journal of Nursing. 2008;21(54):9-24.

37. Mirabzadeh A, Dolatian M, Forouzan AS, Sajjadi H, Majd HA, Mahmoodi Z. Path analysis associations between perceived social support, stressful life events and other psychosocial risk factors during pregnancy and preterm delivery. Iran Red Crescent Med J. 2013;15(6):507-514. doi:10.5812/ircmj.11271

38. Momeni Javid F, Simbar M, Dolatian M, Alavi Majd $\mathrm{H}$. Comparison of lifestyles of women with gestational diabetes and healthy pregnant women. Glob J Health Sci. 2014;7(2):162-169. doi:10.5539/gjhs.v7n2p162

39. Mazlom SR, Darban F, Vaghei S, Modarres gharavi M, Kashani lotfabadi M, Shad M. The effect of Stress Inoculation Program (SIP) on nurses' Perceived stress in psychiatric wards. Evidence Based Care. 2012;2(1):35-44. doi:10.22038/ebcj.2012.388

40. Sarason IG, Levine HM, Basham RB, Sarason BR. Assessing social support: The Social Support Questionnaire. J Pers Soc Psychol.1983;44(1):127-139. doi:10.1037/00223514.44.1.127

41. Nasseh M, Ghazinour M, Joghataei MT, Nojomi M, Richter J. A Persian Version of the Social Support Questionnaire (SSQ). Social Welfare Quarterly. 2011;11(41):251-266.

42. Sararoudi RB, Sanei H, Baghbanian A. The relationship between type $\mathrm{D}$ personality and perceived social support in myocardial infarction patients. J Res Med Sci. 2011;16(5):627-633.

43. Ekback M, Benzein E, Lindberg M, Arestedt K. The Swedish version of the multidimensional scale of perceived social support (MSPSS)--a psychometric evaluation study in women with hirsutism and nursing students. Health Qual Life Outcomes. 2013;11:168. doi:10.1186/1477-752511-168

44. Zhou K, Li H, Wei X, et al. Reliability and validity of the multidimensional scale of perceived social support in Chinese mainland patients with methadone maintenance treatment. Compr Psychiatry. 2015;60:182-188. doi:10.1016/j.comppsych.2015.03.007

45. Jaksic N, Ivezic E, Jokic-Begic N, Suranyi Z, StojanovicSpehar S. Factorial and diagnostic validity of the Beck Depression Inventory-II (BDI-II) in Croatian primary health care. J Clin Psychol Med Settings. 2013;20(3):311322. doi:10.1007/s10880-013-9363-2

46. Hall BJ, Hood MM, Nackers LM, Azarbad L, Ivan I, Corsica J. Confirmatory factor analysis of the Beck Depression Inventory-II in bariatric surgery candidates. Psychol Assess. 2013;25(1):294-299. doi:10.1037/a0030305

47. Ghassemzadeh H, Mojtabai R, Karamghadiri N, Ebrahimkhani N. Psychometric properties of a Persianlanguage version of the Beck Depression Inventory-Second edition: BDI-II-PERSIAN. Depress Anxiety. 2005;21(4):185-192. doi:10.1002/da.20070

48. Dorosty Motlagh AR, Nooraliey P, Sadrzadeh-Yeganeh H, Hosseini M, Karimi J. Determination of Economic - Social factors associated with weight status and depression and the 
relationship between obesity and depression with regard to these factors in high school girls. Payesh. 2015;14(3):305313.

49. Moshki M, Baloochi Beydikhti T, Cheravi K. The Relationship of Postpartum Depression to Health Control Beliefs and Demographic Factors. J Zanjan Univ Med Sci. 2014;22(92):74-85.

50. Aeenparast A, Farzadi F, Maftoon F, et al. Depression among the general population in Iran: Iranian health perception survey. Payesh. 2012;11(2):221-226.

51. Ahmadvand A, Sepehrmanesh Z, Ghoreishi FS, Afshinmajd S. Prevalence of psychiatric disorders in the general population of Kashan, Iran. Arch Iran Med. 2012;15(4):205209. doi:012154/aim.006

52. Miech RA, Shanahan MJ. Socioeconomic status and depression over the life course. J Health Soc Behav. 2000;41(2):162-176. doi:10.2307/2676303

53. Kosidou K, Dalman C, Lundberg M, Hallqvist J, Isacsson G, Magnusson C. Socioeconomic status and risk of psychological distress and depression in the Stockholm Public Health Cohort: a population-based study. J Affect Disord. 2011;134(1-3):160-167. doi:10.1016/j. jad.2011.05.024

54. Abdollahi F, Zarghami M, Azhar MZ, Sazlina SG, Lye MS. Predictors and incidence of post-partum depression: a longitudinal cohort study. J Obstet Gynaecol Res. 2014;40(12):2191-2200. doi:10.1111/jog.12471

55. Murata C, Kondo K, Hirai H, Ichida Y, Ojima T. Association between depression and socio-economic status among community-dwelling elderly in Japan: the Aichi Gerontological Evaluation Study (AGES). Health Place. 2008;14(3):406-414. doi:10.1016/j.healthplace.2007.08.007

56. Tomczak-Witych A. [The level of social support parameters in relation to coping with stress caused by a disease among female patients suffering from depression]. Psychiatr Pol. 2006;40(3):503-514.

57. Ezzati A, Nouri R, Hasani J. Structural Relationship Model between Social Support, Coping Strategies, Stigma and Depression in Infertile Women in Tehran, Iran, 2010. The Iranian Journal of Obstetrics, Gynecology and Infertility.

\section{3;16(45):20-28.}

58. Seeman TE. Social ties and health: the benefits of social integration. Ann Epidemiol. 1996;6(5):442-451.

59. Wilkinson R, Marmot M. Social determinants of health: the solid facts. World Health Organization; 2003.

60. Wang X, Cai L, Qian J, Peng J. Social support moderates stress effects on depression. Int J Ment Health Syst. 2014;8(1):41. doi:10.1186/1752-4458-8-41

61. He F, Zhou Q, Li J, Cao R, Guan H. Effect of social support on depression of internet addicts and the mediating role of loneliness. Int J Ment Health Syst. 2014;8:34. doi:10.1186/1752-4458-8-34

62. Spoozak L, Gotman N, Smith MV, Belanger K, Yonkers KA. Evaluation of a social support measure that may indicate risk of depression during pregnancy. J Affect Disord. 2009;114(1-3):216-223. doi:10.1016/j.jad.2008.07.015

63. 63- Latendresse G. The interaction between chronic stress and pregnancy: preterm birth from a biobehavioral perspective. J Midwifery Womens Health. 2009;54(1):8-17. doi:10.1016/j.jmwh.2008.08.001

64. Booij SH, Bos EH, Bouwmans ME, et al. Cortisol and alpha-Amylase Secretion Patterns between and within Depressed and Non-Depressed Individuals. PLoS One. 2015;10(7):e0131002. doi:10.1371/journal.pone.0131002

65. Kader Maideen SF, Sidik SM, Rampal L, Mukhtar F. Prevalence, associated factors and predictors of depression among adults in the community of Selangor, Malaysia. PLoS One. 2014;9(4):e95395. doi:10.1371/journal.pone.0095395

66. Kiecolt-Glaser JK, Speicher CE, Holliday JE, Glaser R. Stress and the transformation of lymphocytes by EpsteinBarr virus. J Behav Med. 1984;7(1):1-12.

67. Mehnert A, Lehmann C, Graefen M, Huland H, Koch U. Depression, anxiety, post-traumatic stress disorder and health-related quality of life and its association with social support in ambulatory prostate cancer patients. Eur J Cancer Care (Engl). 2010;19(6):736-745. doi:10.1111/ j.1365-2354.2009.01117.x

68. Hammen C. Stress and depression. Annu Rev Clin Psychol. 2005;1:293-319.

(C) 2018 The Author (s); This is an open-access article distributed under the terms of the Creative Commons Attribution License (http://creativecommons.org/licenses/by/4.0), which permits unrestricted use, distribution, and reproduction in any medium, provided the original work is properly cited. 